\title{
Mechanism of Mycolic Acid Cyclopropane Synthase: A Theoretical Study ${ }^{\dagger}$
}

\author{
Rong-Zhen Liao, ${ }^{\star}$ Polina Georgieva, ${ }^{\ddagger}$ Jian-Guo Yu, ${ }^{\S}$ and Fahmi Himo*, \\ ${ }^{\ddagger}$ Department of Organic Chemistry, Arrhenius Laboratory, Stockholm University, SE-10691 Stockholm, Sweden, and ${ }^{\S}$ College of \\ Chemistry, Beijing Normal University, Beijing 100875, People's Republic of China
}

Received September 15, 2010; Revised Manuscript Received December 15, 2010

\begin{abstract}
The reaction mechanism of mycolic acid cyclopropane synthase is investigated using hybrid density functional theory. The direct methylation mechanism is examined with a large model of the active site constructed on the basis of the crystal structure of the native enzyme. The important active site residue Glu140 is modeled in both ionized and neutral forms. We demonstrate that the reaction starts via the transfer of a methyl to the substrate double bond, followed by the transfer of a proton from the methyl cation to the bicarbonate present in the active site. The first step is calculated to be rate-limiting, in agreement with experimental kinetic results. The protonation state of Glu140 has a rather weak influence on the reaction energetics. In addition to the natural reaction, a possible side reaction, namely a carbocation rearrangement, is also considered and is shown to have a low barrier. Finally, the energetics for the sulfur ylide proposal, which has already been ruled out, is also estimated, showing a large energetic penalty for ylide formation.
\end{abstract}

Mycobacterium tuberculosis mycolic acid cyclopropane synthases (MACSs) catalyze the cyclopropanation of the double bond of unsaturated mycolic acids at proximal and distal positions, using $S$-adenosyl-L-methionine (SAM or AdoMet) as the methyl donor $(I)$. The mycolic acid products are lipid components of the cell envelope of mycobacteria (2). This chemical modification is significant for the pathogenicity, persistence, and virulence of $M$. tuberculosis, making these enzymes therapeutic targets for antibacterial drugs, like isoniazid and ethionamide $(3,4)$.

Four members of the MACS family have been identified, namely, CmaA1, CmaA2, MmaA2, and PcaA $(1 b-1 e)$. These enzymes were found to have different selectivities. CmaAl was found to be responsible for cis cyclopropanation at the distal position of $\alpha$-mycolate $(1 b)$, while CmaA2 was shown to be involved in trans cyclopropanation at the proximal position of oxygenated mycolate (1d). MmaA2 is required for cis cyclopropanation at the distal position of $\alpha$-mycolate or at the proximal position of oxygenated mycolate (1e). PcaA catalyzes cis cyclopropanation at the proximal position of $\alpha$-mycolate (1c). The crystal structures of $\mathrm{CmaA1}, \mathrm{CmaA} 2$, and PcaA have been determined, and they revealed $>50 \%$ identity in their primary sequences, indicating a conserved reaction mechanism (5). In the structure of CmaAl in complex with the product $S$-adenosyl-L-homocysteine (SAH) and didecyldimethylammonium bromide (DDDMAB) (Figure 1), the inhibitor DDDMA cation adopts a U shape and is inserted into the binding pocket. Several aromatic residues, such as Tyr 16, Tyr33, and Tyr232, are thought to provide stabilization to the positive charge of the ammonium through cation $-\pi$ interactions (5). A bicarbonate ion is located close to the ammonium cation and hydrogen-bonded to Glu140, His167, and Tyr232 and the Ser34-Cys35 peptide (5).

\footnotetext{
${ }^{\top}$ This work was supported by The Swedish Research Council (Grants 621-2009-4736 and 622-2009-371 to F.H.) and the National Natural Science Foundation of China (Grants 20733002 and 20873008 to J.-G.Y.).

*To whom correspondence should be addressed. Telephone: +46-8161094. Fax: +46-8-154908. E-mail: himo@organ.su.se.
}

There is another type of bacterial cyclopropane synthase, namely Escherichia coli cyclopropane fatty acid synthase (CFAS), which uses unsaturated phospholipids as substrates (6). CFAS shares up to $33 \%$ sequence identity with MACS and also harbors a bicarbonate ion in the active site, suggesting a similar catalytic mechanism (7).

A direct methyl transfer reaction mechanism for the enzymatic cyclopropanation has been proposed (Scheme 1) (8) on the basis of the crystal structure (5) and also on the basis of fluorine substitution $(8 g)$, onium chalcogen effect $(8 i)$, and deuterium isotope effect ( $8 i$ ) studies. In the first step, the transfer of a methyl from AdoMet to the substrate double bond occurs to form a carbocation intermediate. Then the bicarbonate ion acts as a general base to take a proton from the methyl group, resulting in ring closure. Mutations of three active site residues of $E$. coli CFAS (E239, H266, and Y317, corresponding to E140, H167, and $\mathrm{Y} 232$ in CmaA1, respectively), which form hydrogen bonds to the bicarbonate, result in a dramatic decrease in activity (7). This observation might confirm the role of the bicarbonate in the reaction. The first step is suggested to be rate-limiting on the basis of the onium chalcogen effect study, in which the AdoMet was substituted with SeAdoMet and TeAdoMet and different turnover numbers were obtained for the three different methyl donors (8i). In addition, substitution of the methyl donor with deuterium shows an inverse isotope effect, further confirming the suggestion that the methyl transfer step is rate-limiting (8i).

Previously, a metal-assisted sulfur ylide mechanism was suspected, in which the reaction starts by deprotonation of the methyl substituent of AdoMet $(8 a, 8 b)$. However, this has subsequently been ruled out $(8 e)$.

In this study, we use density functional theory (DFT) calculations to shed more light on the reaction mechanism of MACS. A large model of the active site is designed on the basis of the crystal structure of CmaAl. The protein surrounding is modeled by homogeneous continuum model. Recent studies of three different kinds of enzymes have shown that at a model size of $\sim 150-200$ atoms, the solvation effects almost vanish and the choice of 


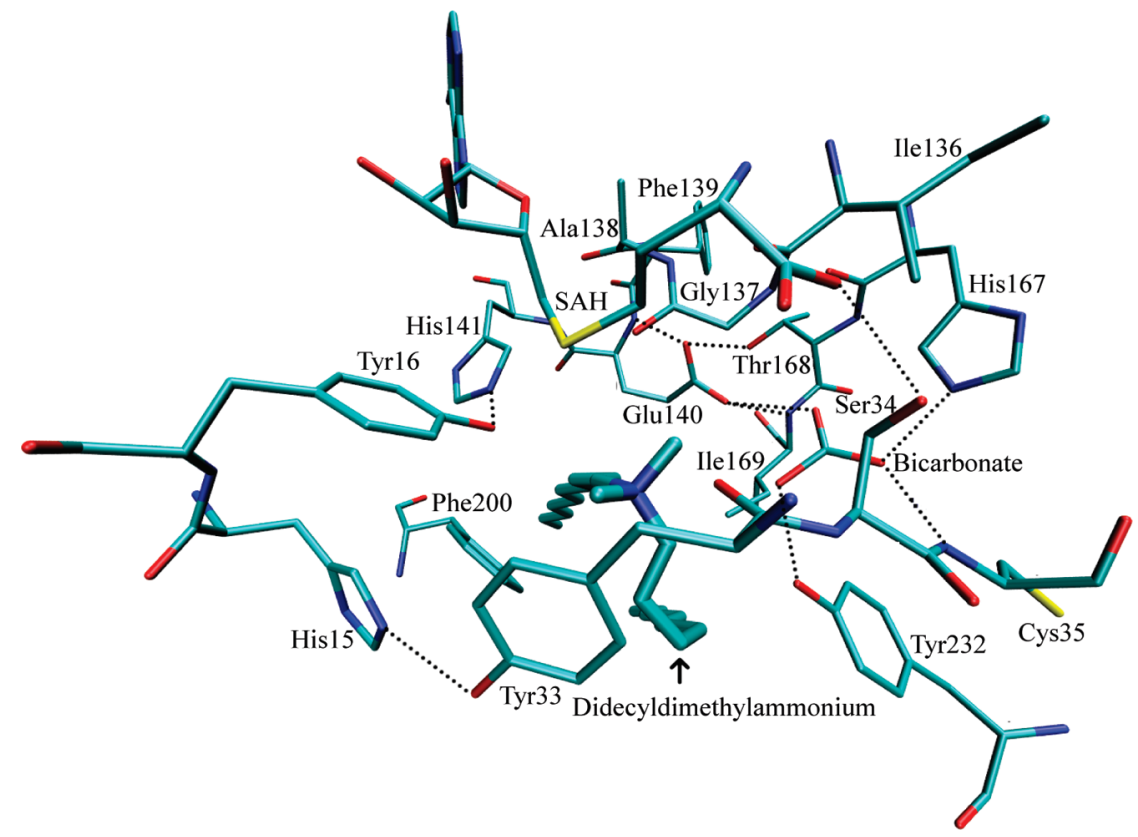

FIgURE 1: X-ray structure of the active site of CmaA1 in complex with SAH and the DDDMA inhibitor (coordinates taken from Protein Data Bank entry $1 \mathrm{KPH})(5)$.
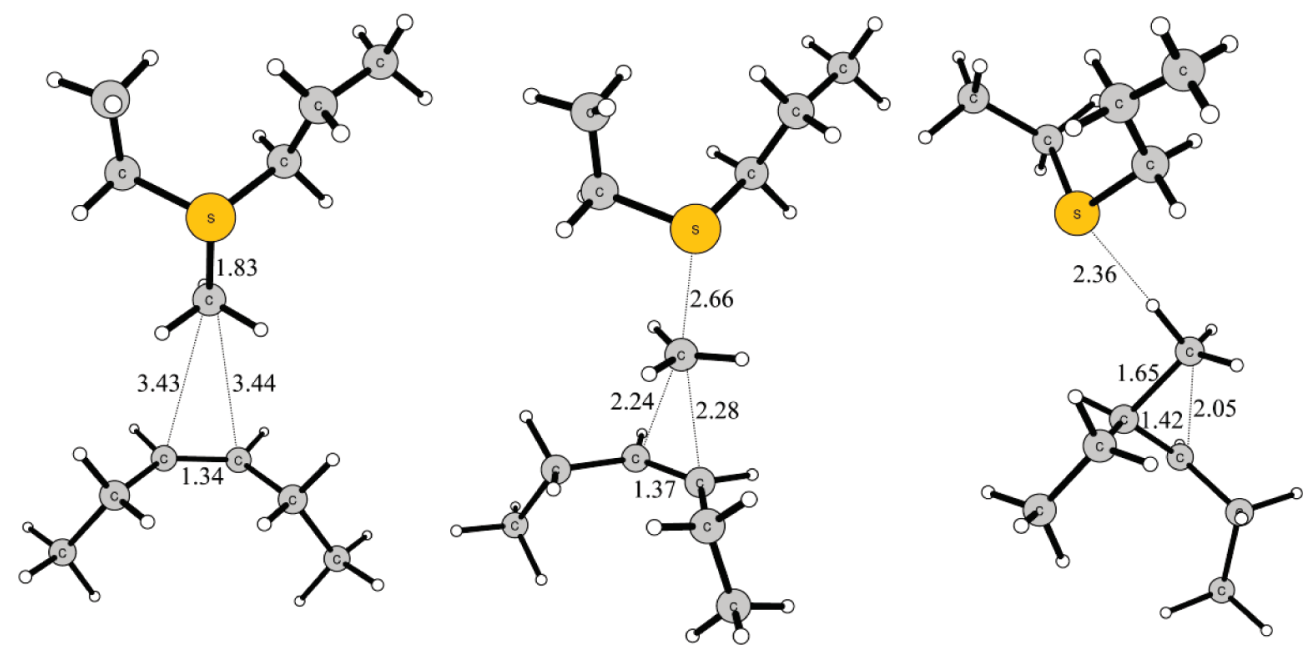

FIGURE 2: Optimized structures of the reactant, transition state, and carbocation intermediate for the nonenzymatic methyl transfer reaction. Distances are in angstroms.

Scheme 1: Proposed Reaction Mechanism for Ring Formation in Cyclopropane Synthase

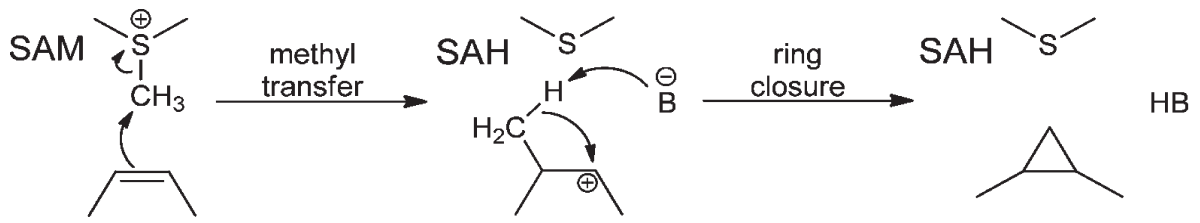

dielectric constant becomes less important (9). This methodology, called the cluster approach, has been successfully applied in studying a wide spectrum of enzymes (10), including a number of methyltransferases (11). The results reported here can be extended to the other members of the MACS and CFAS families.

\section{COMPUTATIONAL DETAILS}

All calculations were performed employing the DFT functional B3LYP (12) as implemented in Gaussian03 (13). Geometry optimizations were conducted using the standard double- $\zeta$ plus polarization $6-31 \mathrm{G}(\mathrm{d}, \mathrm{p})$ basis set. On the basis of these optimized geometries, final energies were evaluated with the larger $6-311+G(2 d, 2 p)$ basis set. To estimate the polarization effects of the surrounding protein environment, single-point calculations were performed at the same level of theory as the geometry optimizations using the conductor-like polarizable continuum model (CPCM) method (14) with the default UA0 radii (united atom topological model). The dielectric constant was chosen to be 4 , a value that has been used in many previous 
applications $(9-11)$. For the small nonenzymatic model (see below), frequency calculations were performed at the same theory level as the geometry optimizations to obtain zero-point energies (ZPE) and to confirm the nature of stationary points. In the active site model, however, the size of the system (more than 180 atoms) prohibited the calculation of frequencies. However, because ZPE effects for the methylation step in the nonenzymatic model are calculated to be less than $1 \mathrm{kcal} / \mathrm{mol}$, the omission of these in the large model is justified. In addition, to preserve the structural integrity of the large active site cluster model, certain atoms were kept fixed to their crystallographic positions during the geometry optimizations (see below). Apart from these centers, the geometries were fully optimized, including those of transition states.

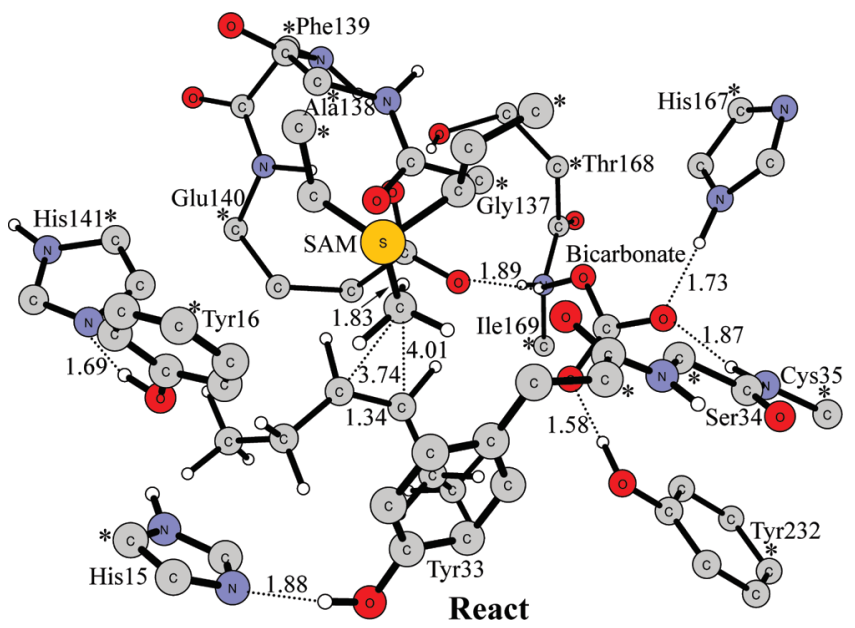

FIGURE 3: Optimized structure of the active site model with ionized Glu140 (React). Asterisks denote atoms that are kept fixed to their crystallographically observed positions in the geometry optimization. For the sake of clarity, some hydrogen atoms are not shown. Distances are given in angstroms.

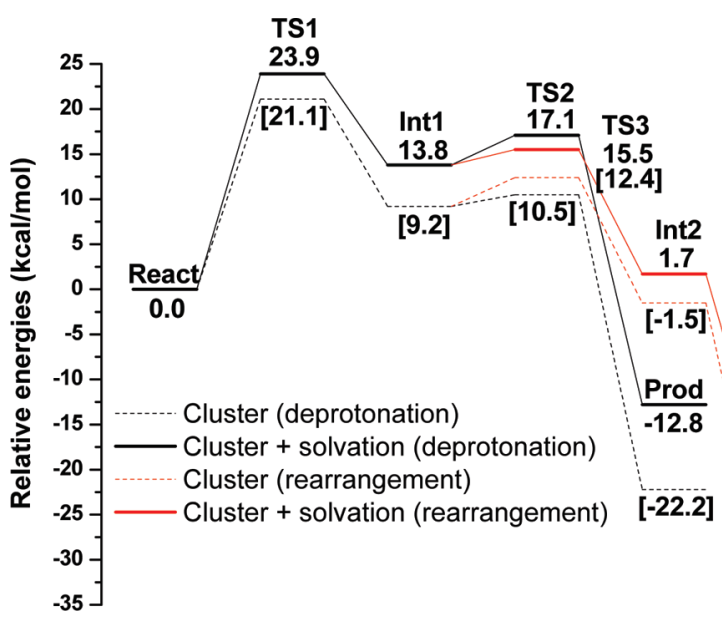

Prod2 $-24.7$

FiguRE 5: Calculated potential energy profile for MACS-catalyzed cyclopropane formation with ionized Glu 140 (black line for carbocation deprotonation and red line for carbocation rearrangement). Values in brackets are without solvation effects.
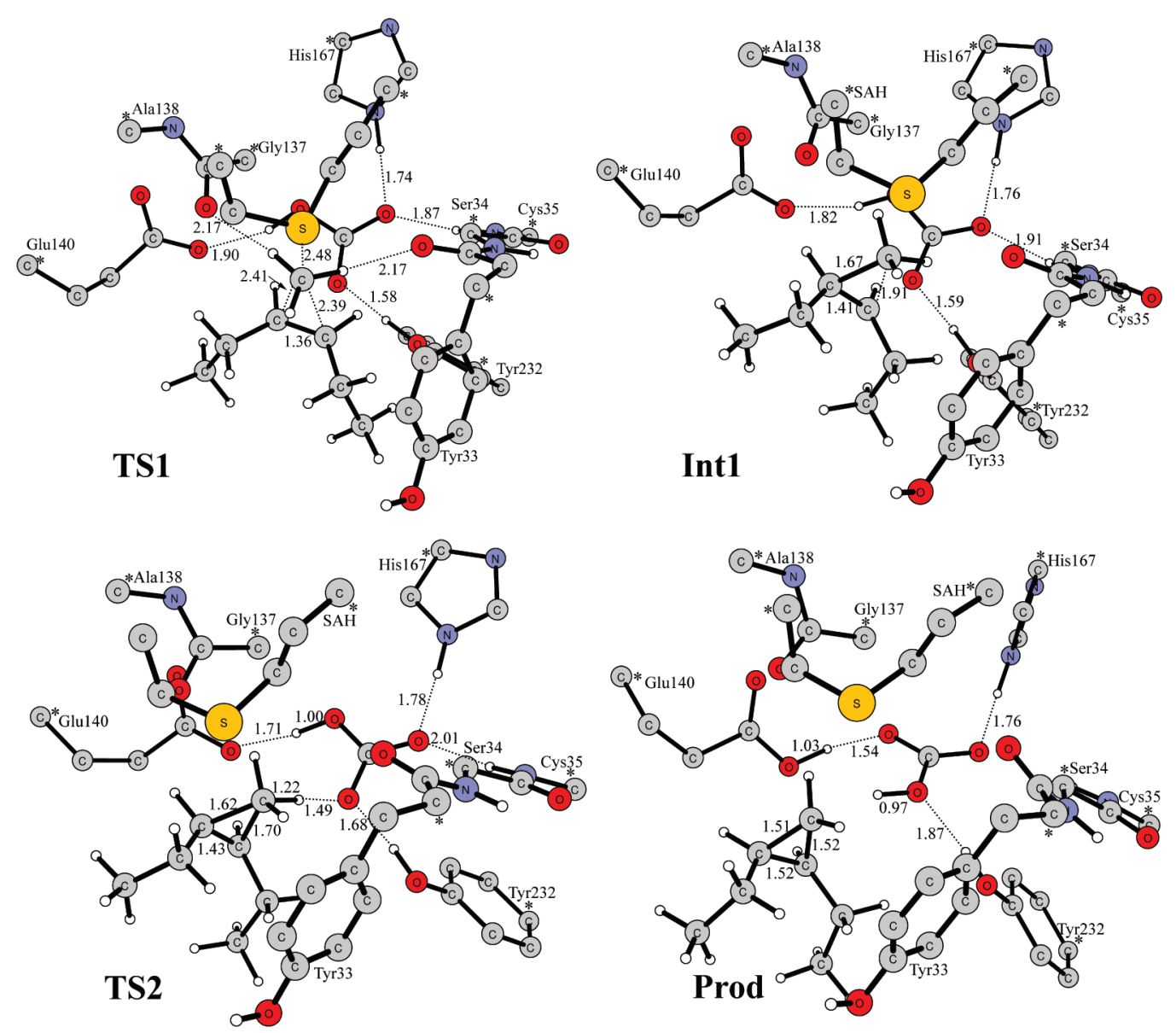

FIgURE 4: Optimized structures of stationary points along the reaction pathway with ionized Glu140. For the sake of clarity, His15, Tyr16, His 141, the Thr168-Ile169 peptide, part of the Ala138-Phe139-Glu140 peptide, and some hydrogen atoms are not shown. 

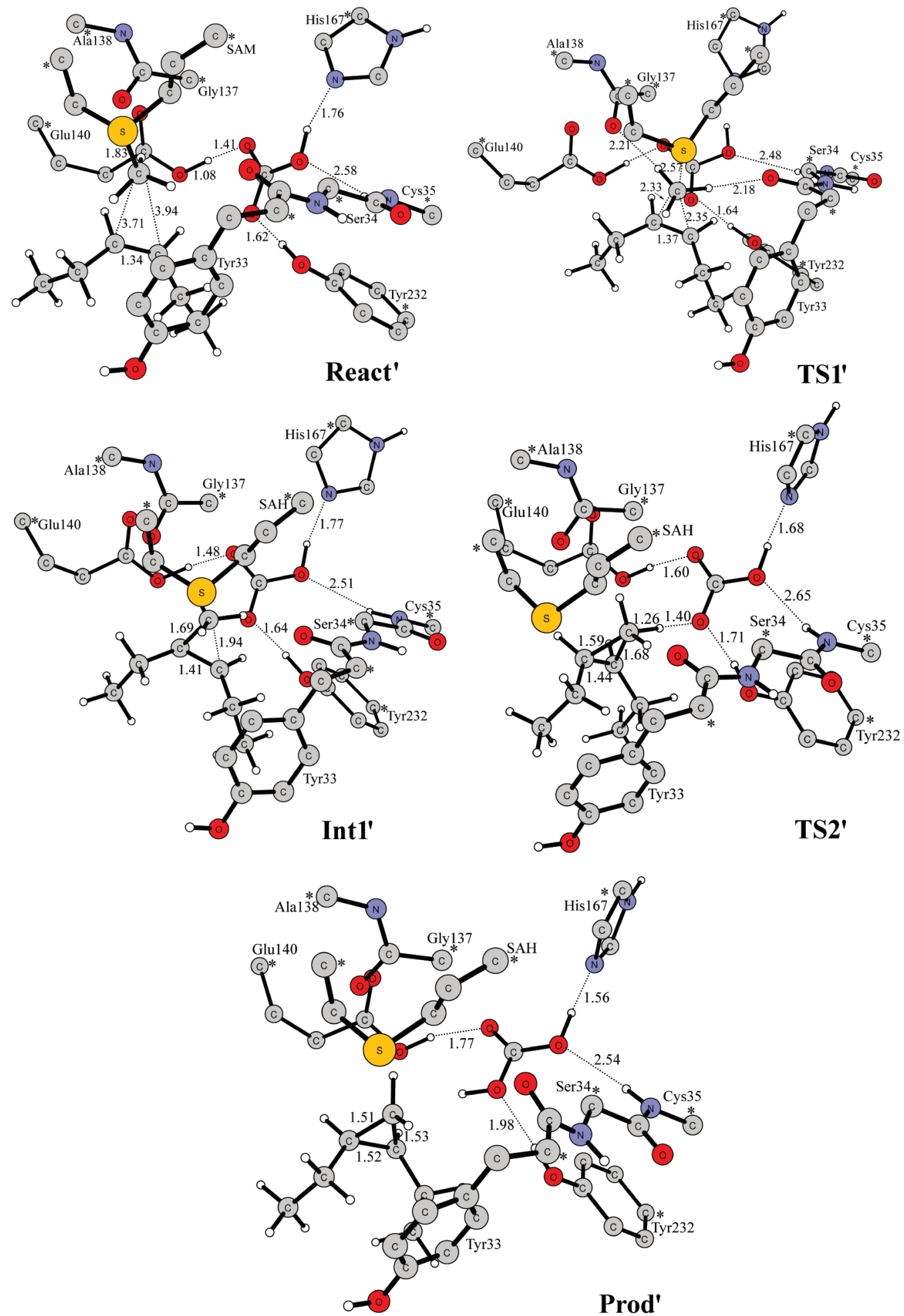

FIGURE 6: Optimized structures of stationary points along the reaction pathway with neutral Glu140.

\section{RESULTS AND DISCUSSION}

Model of the Nonenzymatic Reaction. First, we designed a small model consisting of a truncated sulfonium cation that mimics the SAM cofactor, and a 3-hexene as a model of the substrate, to study the nonenzymatic methyl transfer reaction. No surrounding residues are included, and the model consists of 40 atoms with a total charge of +1 . The comparison between this model and the active site model will be helpful in the analysis of the effects of the various parts in the enzyme active site. The optimized structures of the reactant complex, transition state, and the carbocation intermediate complex are shown in Figure 2. The methyl transfer is calculated to occur through an $\mathrm{S}_{\mathrm{N}} 2$ mechanism. The barrier is calculated to be $26.6 \mathrm{kcal} / \mathrm{mol}$, which increases to $28.7 \mathrm{kcal} / \mathrm{mol}(\varepsilon=4)$ and $29.2 \mathrm{kcal} / \mathrm{mol}(\varepsilon=80)$ upon inclusion of solvation effects. The reaction is calculated to be endothermic by $18.7 \mathrm{kcal} / \mathrm{mol}$ without solvation effects, and by 19.9 and $20.1 \mathrm{kcal} / \mathrm{mol}$ with $\varepsilon$ values of 4 and 80 , respectively. At the transition state (TS), the critical $\mathrm{S}_{\mathrm{D}}-\mathrm{C}$ distance is $2.66 \AA$ and the methyl group is close to planar (improper $\mathrm{H}-\mathrm{C}-\mathrm{H}-\mathrm{H}$ dihedral angle of $159^{\circ}$ ). The distances between the methyl carbon 
and the two carbons of the double bond are 2.42 and $2.28 \AA$. Downhill from the transition state, a carbocation intermediate was located with the methyl group bound asymmetrically to the double bond. The distances between the methyl carbon and the two carbons are now 1.65 and $2.05 \AA$, respectively. This asymmetric feature has also been observed previously for other methyl-bridged carbocations at both the B3LYP and MP2 levels of theory (15). The symmetric bridging fashion, namely a protonated cyclopropane ring, has also been considered. However, its energy is slightly higher $(\sim 1 \mathrm{kcal} / \mathrm{mol})$, and it turns out to be a transition state that connects the two asymmetric forms.

The results of this small model show thus that the enzyme environment has to lower the barrier for the methyl transfer by $\sim 10 \mathrm{kcal} / \mathrm{mol}$. Also, the enzyme has to provide a suitable base that abstracts the proton to complete the reaction.

Model of the Active Site. A large model of the active site was devised on the basis of the X-ray crystal structure of CmaAl in a complex with SAH and the DDDMAB inhibitor [Protein Data Bank (PDB) entry 1KPH (5)]. The model contains SAH, the bicarbonate ion $\left(\mathrm{HCO}_{3}{ }^{-}\right)$, Tyr33-Ser34-Cys35, Gly137-Ala138Phe139-Glu140, and Thr168-Ile169 backbones, and truncated models of the side chains of His15, Tyr16, Tyr33, Glu140, His141, His167, Thr168, and Tyr232 (Figure 3). SAH was converted to SAM via addition of a methyl group to sulfur, and it was truncated with two and three carbons on each side of the sulfur center, a case similar to the model used in the nonenzymatic reaction described above. On the basis of previous studies, this model of SAM has been shown to adequately describe the $\mathrm{S}-\mathrm{C}$ bond and to have sufficient flexibility to accommodate structural changes taking place during the reaction $(9 c, 11)$. All residues are truncated such that only important side chains or peptide backbones are included in the model (see Figure 3). Hydrogen atoms were added manually. The important Glu140 residue is hydrogen-bonded to the bicarbonate and has a significant influence on the deprotonation step. As its protonation state is not established, this residue is modeled in both the ionized and neutral forms. To preserve the structural integrity of the cluster model, the truncation atoms were kept fixed to their crystallographic positions during the geometry optimizations. These atoms are marked with asterisks in the figures below. As a substrate, we used 3-hexene as in the nonenzymatic model. The model with an ionized Glu140 consists of 181 atoms with a total charge of -1 , while with a neutral Glu140 it consists of 182 atoms with a total charge of 0 .

Methylation with Ionized Glu140. In the reactant structure (React, Figure 3), the hydrogen bonding network between the bicarbonate and the Ser34-Cys35 peptide, Glu140, His167, and Tyr232 is well-reproduced in the model compared to the crystal structure. No significant interaction is observed between the 3-hexene substrate and its surrounding residues. The methyl group to be transferred is well-situated on the top of the substrate double bond. The distances between the methyl carbon and the two double bond carbons are 3.74 and $4.01 \AA$. Similar to those of other methyltransferases studied previously, the methyl transfer is found to take place via an $\mathrm{S}_{\mathrm{N}} 2$ mechanism $(9 c, 11)$. The optimized transition state for this step (TS1) and the resulting carbocation intermediate (Int1) are shown in Figure 4. The barrier is calculated to be $23.9 \mathrm{kcal} / \mathrm{mol}(21.1 \mathrm{kcal} / \mathrm{mol}$ without solvation), and Int1 lies at $13.8 \mathrm{kcal} / \mathrm{mol}(9.2 \mathrm{kcal} / \mathrm{mol}$ without solvation) relative to React. The barrier is thus $\sim 5 \mathrm{kcal} / \mathrm{mol}$ lower than that of the nonenzymatic model with an $\varepsilon$ of 4 . At TS1, the critical S-C distance is $2.48 \AA$, which is somewhat shorter than in

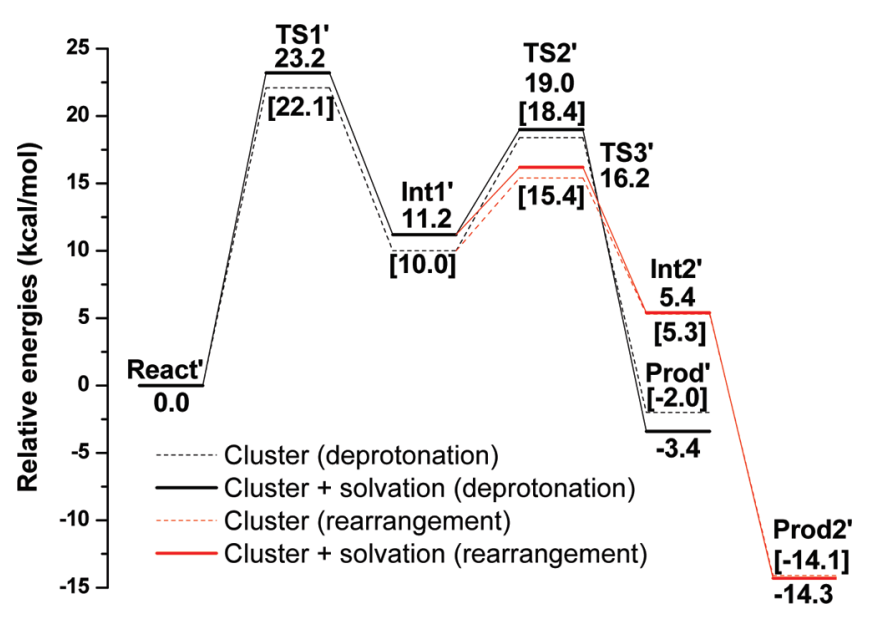

FIGURE 7: Calculated potential profile for MACS-catalyzed cyclopropane formation with neutral Glu140 (black line for carbocation deprotonation and red line for carbocation rearrangement). Values in brackets are without solvation effects.

the nonenzymatic model $(2.66 \AA)$ but is slightly longer than the corresponding distance in $N$-methyltransferases $(9 c, 11)$. The distances between the methyl carbon and the two double bond carbons are now 2.41 and $2.39 \AA$ A. The Gly137-Ala138 and Tyr33Ser34 peptide oxygens interact with the transferred methyl group, with $\mathrm{O} \cdots \mathrm{H}$ distances of $2.17 \AA$. These interactions provide stabilization to the methyl carbocation in TS1 and contribute thus to the lowering of the barrier.

Downhill from this transition state, the formed Int1 carbocation is calculated to be asymmetric. The bond distances between the methyl carbon and the two carbons are 1.67 and $1.91 \AA$, which are similar to those of the nonenzymatic model (1.65 and $2.05 \AA$, respectively). At Int1, we can see that the anionic bicarbonate is quite close to the carbocation $(\mathrm{O} \cdots \mathrm{C}$ distance of $2.94 \AA$ ). It provides electrostatic stabilization to the carbocation intermediate and also assists in lowering the barrier. In addition, the nearby Tyr33 residue might provide $\pi$-cation interaction to stabilize the carbocation intermediate (16).

In the following step, three events are seen to take place concertedly, namely, the transfer of a proton from the methyl group of the carbocation to the bicarbonate, the transfer of a proton from bicarbonate to Glu140, and ring closure to form the cyclopropane product. The optimized transition state (TS2) is shown in Figure 4. The barrier is calculated to be only $3.3 \mathrm{kcal} /$ mol from Int1. According to this, the bicarbonate functions as a bridge to shuttle the proton from the carbocation to Glu140, which can be considered as the base in this step. The energy of the product complex (Prod) is calculated to be $11.7 \mathrm{kcal} / \mathrm{mol}$ lower than that of React $(22.1 \mathrm{kcal} / \mathrm{mol}$ without solvation). The large solvation effect observed is due to the charge separation in React ( +1 on SAM and -1 each on Glu140 and bicarbonate), compared to Prod (neutral SAH, neutral Glu140, and -1 on only bicarbonate).

The potential energy profile for formation of the cyclopropane with the ionized Glu140 model is shown in Figure 5. From this, one can see that the first step is rate-limiting, in accordance with the onium chalcogen effect experiments $(8 i)$. Experimental rate constants have been measured to be in the range of $2-12 \min ^{-1}(7 \mathrm{~b}, 8 \mathrm{~h}, 8 \mathrm{i})$, which can be converted to barriers of around $19 \mathrm{kcal} / \mathrm{mol}$ using classical transition state theory. The calculated barrier of $23.9 \mathrm{kcal} / \mathrm{mol}$ is thus in quite good agreement, but 
Liao et al.

somewhat overestimated. Similar behavior was also seen in a previous study of the mechanism of methylguanine-DNA methyltransferase (11d).

Scheme 2: Possible Carbocation Rearrangement<smiles>C/C=C(/C)C(C)=C(C)C(C)C(C)C</smiles>
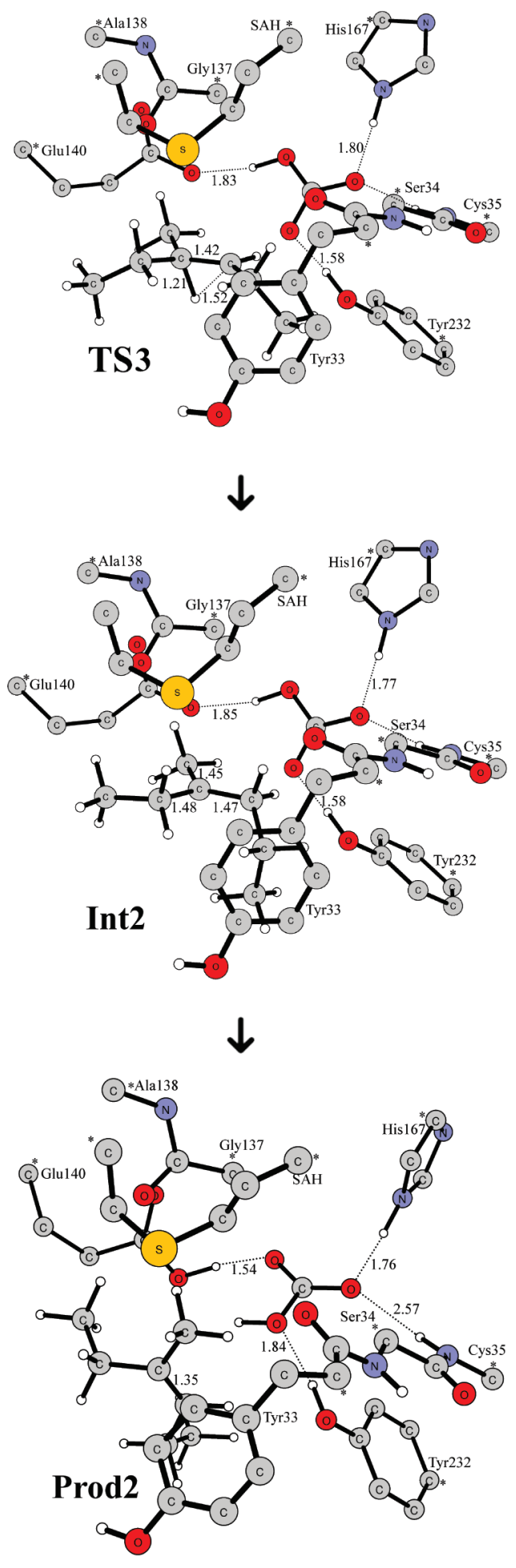

Methylation with Neutral Glu140. The calculations described above assume that the Glu140 residue is in the ionized form and acts as a general base to take a proton from the bicarbonate during the deprotonation of the carbocation intermediate. However, it can be envisioned that this residue instead is in the neutral form. This could lead to different energetics, especially for the deprotonation step. We have therefore investigated the reaction mechanism for this scenario.

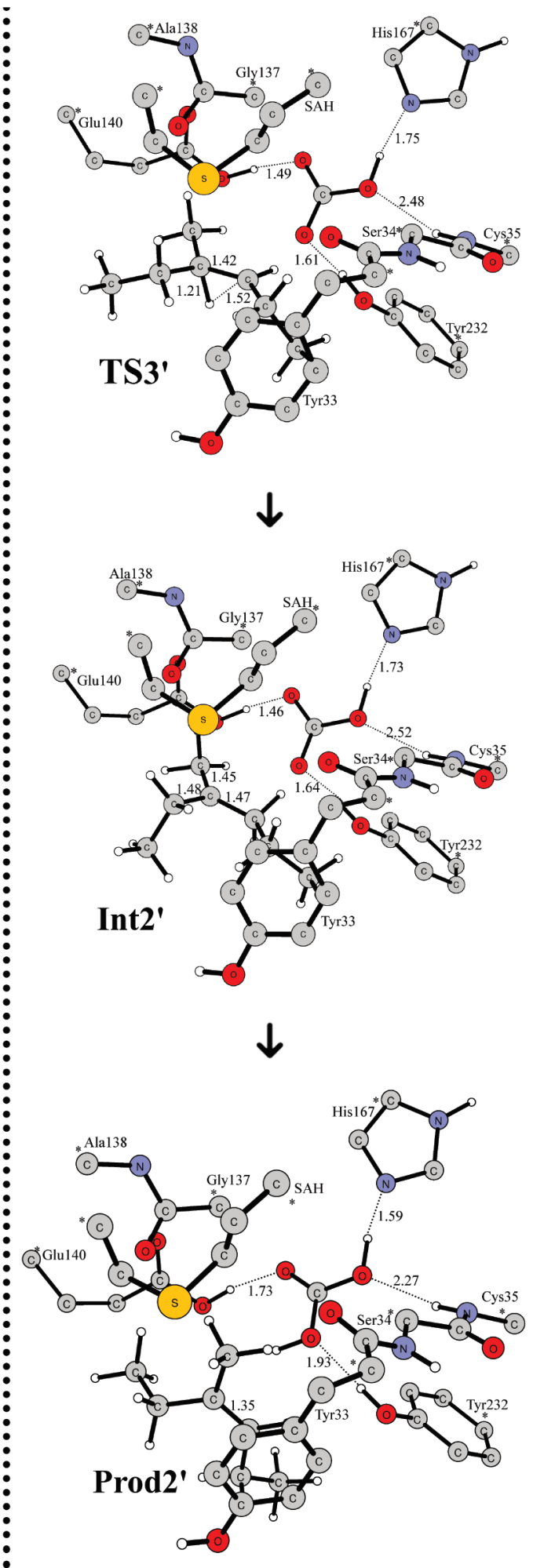

FIGURE 8: Optimized structures for the transition states, intermediates, and products for carbocation rearrangement with both ionized (left) and neutral (right) Glu140. 
In the reactant complex (called React' in Figure 6), the negatively charged bicarbonate now is donating a hydrogen bond to His 167 and accepting a hydrogen bond from the neutral Glu140. The optimized structures of the transition state for the methyl transfer $\left(\mathbf{T S 1}^{\prime}\right)$ and the resulting carbocation intermediate (Int1') are also displayed in Figure 6. The critical geometric parameters of $\mathbf{T S 1}^{\prime}$ are quite similar to those of TS1 with ionized Glu140. The $\mathrm{S}-\mathrm{C}$ distance is $2.57 \AA$, slightly longer than that in TS1, indicating slightly later transition state when the Glu140 residue is neutral. Correspondingly, the distances between the transferred methyl carbon and the two double bond carbons become slightly shorter ( 2.33 and $2.35 \AA)$. The barrier is calculated to be $23.2 \mathrm{kcal} / \mathrm{mol}$ ( $22.1 \mathrm{kcal} / \mathrm{mol}$ without solvation), which is very similar to that with ionized Glu1 $40(23.9 \mathrm{kcal} / \mathrm{mol})$. The resulting carbocation intermediate Int1' lies at $11.2 \mathrm{kcal} / \mathrm{mol}$ relative to React', also quite similar to Int1.

In the next step, the bicarbonate takes a proton from the methyl group, which is concomitant with ring closure. The transition state $\left(\mathbf{T S 2}^{\prime}\right)$ and resulting product $\left(\right.$ Prod $\left.^{\prime}\right)$ were also optimized and are depicted in Figure 6. The barrier now becomes $19.0 \mathrm{kcal} / \mathrm{mol}$ relative to React' ( $18.4 \mathrm{kcal} / \mathrm{mol}$ without solvation), only $\sim 2 \mathrm{kcal} / \mathrm{mol}$ higher than that with ionized Glu140. The reaction now is only slightly exothermic, as the resulting enzyme-product complex (Prod') lies $3.4 \mathrm{kcal} / \mathrm{mol}$ lower than React'. The energies of this scenario are summarized in Figure 7.

As one can see in Figures 5 and 7, both cases, with Glu140 being ionized or neutral, give reasonable and quite similar barriers. In both cases, the first step is calculated to be ratelimiting, with a barrier of $\sim 23 \mathrm{kcal} / \mathrm{mol}$. The deprotonation step has an only slightly higher barrier with neutral Glu140 compared to the ionized one.

Carbocation Rearrangement. From Int1 and Int1', it is conceivable that the carbocation can undergo a rearrangement through a [1,2]-hydride shift, forming a more stable tertiary carbocation intermediate, which then would become deprotonated to form 3-methyl-3-hexene (Scheme 2) (17).

We have here located the transition states for this rearrangement for both cases, i.e., with Glu140 being ionized or neutral. The optimized TSs (called TS3 and $\mathbf{T S 3} \mathbf{3}^{\prime}$ ) together with the resulting tertiary carbocation intermediates (Int2 and Int2') are shown in Figure 8. It turns out that in both cases these rearrangement reactions have somewhat lower barriers compared to those of the deprotonation reactions described above. The obtained energies are inserted in the graphs shown in Figures 5 and 7 for comparison.

For the case of anionic Glu140, the barrier from Int1 is calculated to be $1.7 \mathrm{kcal} / \mathrm{mol}(3.2 \mathrm{kcal} / \mathrm{mol}$ without solvation correction), compared to $3.3 \mathrm{kcal} / \mathrm{mol}(1.3 \mathrm{kcal} / \mathrm{mol}$ without solvation) for the proton transfer (TS2). The subsequent transfer of a proton from the tertiary carbocation Int 2 to the anionic bicarbonate has also a very low barrier (18). Together, these two steps are exothermic by $38.5 \mathrm{kcal} / \mathrm{mol}$, indicating that the rearrangement is irreversible.

For the case of neutral Glu140, the barrier is $5.0 \mathrm{kcal} / \mathrm{mol}(5.4$ $\mathrm{kcal} / \mathrm{mol}$ without solvation) relative to Int1' ${ }^{\prime}$, which is $2.8 \mathrm{kcal} /$ $\mathrm{mol}\left(3.0 \mathrm{kcal} / \mathrm{mol}\right.$ without solvation) lower than TS2 ${ }^{\prime}$. Now, the resulting intermediate $\mathbf{I n t} \mathbf{2}^{\prime}$ is only $5.8 \mathrm{kcal} / \mathrm{mol}$ lower than Int $\mathbf{1}^{\prime}$, and the 3-methyl-3-hexene Prod $2^{\prime}$ is $25.5 \mathrm{kcal} / \mathrm{mol}$ lower that Int1'.

These energies show that the [1,2]-shift is slightly more favored compared to the deprotonation mechanism described in the previous sections. However, the fact that cyclopropane is the sole product observed for the enzymatic reaction indicates that the rearrangement cannot take place in the enzyme. This means that the enzyme somehow must block this side reaction to yield the desired mycolic acid cyclopropane product. Exactly how this is achieved is not clear to us at present.

It is possible that the results are artifacts of the active site model employed in this study. It could, for example, be that the model is not large enough to account for geometric constraints that could raise the barrier of the rearrangement reaction and lower the barrier for proton transfer. It is also possible that it is an error of the theoretical protocol used in this study. However, tests of single-point calculations using two other functionals, namely, BB1K (19) and B3PW91 (20), also gave similar results (21).

Sulfur Ylide Mechanism. Although the sulfur ylide mechanism has been ruled out for MACS on the basis of extensive experimental studies, we still examine this possibility here to compare the energies. Sulfur ylides are widely used for cyclopropanation reactions in organic synthesis (22), where a strong base is used to generate the reactive sulfur ylide $(22 b, 22 e, 23)$. In the active site of MACS, a possible base could be the bicarbonate, even though it does not directly interact with the methyl group. However, bicarbonate is not expected to be sufficiently strong to accomplish this. We have optimized the ylide intermediate structure [Ylide and Ylide' (see the Supporting Information)] with one proton transferred from the methyl group to the bicarbonate ion for both models with ionized and neutral Glu140. Indeed, the energy of Ylide is as much as $36.5 \mathrm{kcal} / \mathrm{mol}$ higher than that of React with ionized Glu140 and $46.6 \mathrm{kcal} / \mathrm{mol}$ with neutral Glu140. Thus, our calculations further confirm that the sulfur ylide mechanism is not energetically viable.

\section{CONCLUSIONS}

In this paper, we employed density functional theory to investigate the reaction mechanism of mycolic acid cyclopropane synthase using a large model of the active site. The energetics for the direct methylation mechanism were examined for the two scenarios in which the important Glu140 active site residue is either ionized or neutral. The calculations support the previously suggested mechanism and provide a detailed characterization of the reaction pathway.

Cyclopropane formation proceeds through a two-step mechanism: an $\mathrm{S}_{\mathrm{N}} 2$ transfer of a methyl group from SAM to the substrate double bond, followed by the transfer of a proton from the methyl group to bicarbonate coupled with ring closing. The methyl transfer results in a carbocation intermediate, stabilized by mainly electrostatic interactions with the negative charge of the Glu140-bicarbonate diad and two peptide backbone oxygens. The first step is rate-limiting, in agreement with experimental observations. The protonation state of Glu140 is demonstrated to have quite weak effects on the energetics of the reaction.

One interesting observation from the calculations is the fact that the rearrangement of the carbocation intermediate through a [1,2]-shift has a slightly lower barrier than the proton transfer. This would lead to a mixture of products. However, because cyclopropane is the sole product observed experimentally, the enzyme has to block this route somehow. We have also speculated that this result could be an artifact of the employed quantum chemical active site model.

Finally, because of the poor basicity of the bicarbonate, ylide formation via the transfer of a proton from the methyl group to the bicarbonate is shown to be energetically very unfavorable. 


\section{ACKNOWLEDGMENT}

F.H. gratefully acknowledges computer time allocation from the PDC Center for High Performance Computing.

\section{SUPPORTING INFORMATION AVAILABLE}

Optimized structures of the ylide mechanism and Cartesian coordinates of all presented structures. This material is available free of charge via the Internet at http://pubs.acs.org

\section{REFERENCES}

1. (a) Yuan, Y., Mead, D., Schroeder, B. G., Zhu, Y.-Q., and Barry, C. E., III (1998) The biosynthesis of mycolic acids in Mycobacterium tuberculosis: Enzymatic methyl(ene) transfer to acyl carrier protein bound meromycolic acid in vitro. J. Biol. Chem. 273, 21282-21290. (b) Yuan, Y., Lee, R. E., Besra, G. S., Belisle, J. T., and Barry, C. E., III (1995) Identification of a gene involved in the biosynthesis of cyclopropanated mycolic acids in Mycobacterium tuberculosis. Proc. Natl. Acad. Sci. U.S.A. 92, 6630-6634. (c) Glickman, M. S., Cox, J. S., and Jacobs, W. R., Jr. (2000) A novel mycolic acid cyclopropane synthetase is required for cording, persistence, and virulence of Mycobacterium tuberculosis. Mol. Cell 5, 717-727. (d) Glickman, M. S., Cahill, S. M., and Jacobs, W. R., Jr. (2001) The Mycobacterium tuberculosis cmaA2 gene encodes a mycolic acid trans-cyclopropane synthetase. J. Biol. Chem. 276, 2228-2233. (e) Glickman, M. S. (2003) The mmaA2 gene of Mycobacterium tuberculosis encodes the distal cyclopropane synthase of the $\alpha$-mycolic acid. J. Biol. Chem. 278, 7844-7849.

2. (a) Asselineau, C., Asselineau, J., Lanéelle, G., and Lanéelle, M.-A. (2002) The biosynthesis of mycolic acids by Mycobacteria: Current and alternative hypotheses. Prog. Lipid Res. 41, 501-523. (b) Takayama, K., Wang, C., and Besra, G. S. (2005) Pathway to synthesis and processing of mycolic acids in Mycobacterium tuberculosis. Clin. Microbiol. Rev. 18, 81-101.

3. (a) Vilchèze, C., Wang, F., Arai, M., Hazbón, M. H., Colangeli, R. Kremer, L., Weisbrod, T. R., Alland, D., Sacchettini, J. C., and Jacobs, W. R., Jr. (2006) Transfer of a point mutation in Mycobacterium tuberculosis inhA resolves the target of isoniazid. Nat. Med. 12, 1027-1029. (b) Wang, F., Langley, R., Gulten, G., Dover, L. G., Besra, G. S., Jacobs, W. R., Jr., and Sacchettini, J. C. (2007) Mechanism of thioamide drug action against tuberculosis and leprosy. J. Exp. Med. 204, 73-78.

4. Smith, C. V., Sharma, V., and Sacchettini, J. C. (2004) TB drug discovery: Addressing issues of persistence and resistance. Tuberculosis 84, 45-55.

5. Huang, C., Smith, C. V., Glickman, M. S., Jacobs, W. R., Jr., and Sacchettini, J. C. (2002) Crystal structures of mycolic acid cyclopropane synthases from Mycobacterium tuberculosis. J. Biol. Chem. 277, 11559-11569.

6. (a) Cronan, J. E., Jr. (2002) Phospholipid modifications in bacteria. Curr. Opin. Microbiol. 5, 202-205. (b) Bao, X., Katz, S., Pollard, M., and Ohlrogge, J. (2002) Carbocyclic fatty acids in plants: Biochemical and molecular genetic characterization of cyclopropane fatty acid synthesis of Sterculiafoetida. Proc. Natl. Acad. Sci. U.S.A. 99, 71727177.

7. (a) Iwig, D. F., Uchida, A., Stromberg, J. A., and Booker, S. J. (2005) The activity of Escherichia coli cyclopropane fatty acid synthase depends on the presence of bicarbonate. J. Am. Chem. Soc. 127, 11612-11613. (b) Courtois, F., and Ploux, O. (2005) Escherichia coli cyclopropane fatty acid synthase: Is a bound bicarbonate ion the active-site base? Biochemistry 44, 13583-13590.

8. (a) Pohl, S., Law, J. H., and Ryhage, R. (1963) The path of hydrogen in the formation of cyclopropane fatty acids. Biochim. Biophys. Acta 70, 583-585. (b) Cohen, T., Herman, G., Chapman, T. M., and Kuhn, D. (1974) Laboratory model for the biosynthesis of cyclopropane rings. Copper-catalyzed cyclopropanation of olefins by sulfur ylides. J. Am. Chem. Soc. 96, 5627-5628. (c) Lederer, E. (1969) Some problems concerning biological C-alkylation reactions and phytosterol biosynthesis. Q. Rev. Chem. Soc. 23, 453-481. (d) Buist, P. H., and MacLean, D. B. (1981) The biosynthesis of cyclopropane fatty acids. I. Feeding experiments with oleic acid-9,10-d2, oleic acid8,8,11,11-d4, and L-methionine-methyl-d3. Can. J. Chem. 59, 828838. (e) Buist, P. H., and MacLean, D. B. (1982) The biosynthesis of cyclopropane fatty acids. II. Mechanistic studies using methionine labelled with one, two, and three deuterium atoms in the methyl group. Can. J. Chem. 60, 371-378. (f) Buist, P. H., and Pon, R. A.
(1990) An unexpected reversal of fluorine substituent effects in the biomethylenation of two positional isomers: A serendipitous discovery. J. Org. Chem. 55, 6240-6241. (g) Molitor, E. J., Paschal, B. M., and Liu, H.-W. (2003) Cyclopropane fatty acid synthase from Escherichia coli: Enzyme purification and inhibition by vinylfluorine and epoxide-containing substrate analogues. ChemBioChem 4, 13521356. (h) Courtois, F., Guérard, C., Thomas, X., and Ploux, O. (2004) Escherichia coli cyclopropane fatty acid synthase. Eur. J. Biochem. 271, 4769-4778. (i) Iwig, D. F., Grippe, A. T., McIntyre, T. A., and Booker, S. J. (2004) Isotope and elemental effects indicate a rate-limiting methyl transfer as the initial step in the reaction catalyzed by Escherichia coli cyclopropane fatty acid synthase. Biochemistry 43, $13510-13524$

9. (a) Sevastik, R., and Himo, F. (2007) Reaction Mechanism of cisChloroacrylic Acid Dehalogenase: A Theoretical Study. Bioorg. Chem. 35, 444 457. (b) Hopmann, K. H., and Himo, F. (2008) Quantum Chemical Modeling of the Dehalogenation Reaction of Haloalcohol Dehalogenase. J. Chem. Theor. Comput. 4, 1129-1137. (c) Georgieva, P., and Himo, F. (2010) Quantum Chemical Modeling of Enzymatic Reactions: The Case of Histone Lysine Methyltransferase. J. Comput. Chem. 31, 1707-1714.

10. See, for example, the following reviews: (a) Blomberg, M. R. A., and Siegbahn, P. E. M. (2001) A Quantum Chemical Approach to the Study of Reaction Mechanisms of Redox-Active Metalloenzymes. J. Phys. Chem. B 105, 9375-9386. (b) Himo, F., and Siegbahn, P. E. M. (2003) Quantum Chemical Studies of Radical-Containing Enzymes. Chem. Rev. 103, 2421-2456. (c) Noodleman, L., Lovell, T., Han, W.-G., Li, J., and Himo, F. (2004) Quantum Chemical Studies of Intermediates and Reaction Pathways in Selected Enzymes and Catalytic Synthetic Systems. Chem. Rev. 104, 459-508. (d) Siegbahn, P. E. M., and Borowski, T. (2006) Modeling enzymatic reactions involving transition metals. Acc. Chem. Res. 39, 729-738. (e) Himo, F. (2006) Quantum Chemical Modeling of Enzyme Active Sites and Reaction Mechanisms. Theor. Chem. Acc. 116, 232-240. (f) Ramos, M. J., and Fernandes, P. A. (2008) Computational Enzymatic Catalysis. Acc. Chem. Res. 41, 689-698. (g) Himo, F., and Siegbahn, P. E. M. (2009) Recent Developments of the Quantum Chemical Cluster Approach for Modeling Enzyme Reactions. J. Biol. Inorg. Chem. 14, 643-651. (h) Blomberg, M. R. A., and Siegbahn, P. E. M. (2010) Quantum chemistry as a tool in bioenergetics. Biochim. Biophys. Acta 1797, 129-142.

11. (a) Velichkova, P., and Himo, F. (2005) Methyl transfer in glycine N-methyltransferase. A theoretical study. J. Phys. Chem. B 109, 82168219. (b) Velichkova, P., and Himo, F. (2006) Theoretical study of the methyl transfer in guanidinoacetate methyltransferase. J. Phys. Chem. B 110, 16-19. (c) Georgieva, P., Wu, Q., McLeish, M. J., and Himo, F. (2009) The Reaction Mechanism of Phenylethanolamine N-Methyltransferase: A Density Functional Theory Study. Biochim. Biophys. Acta 1794, 1831-1837. (d) Georgieva, P., and Himo, F. (2008) Theoretical Study of the Reaction Mechanism of the DNA Repairing Enzyme Alkylguanine Alkyltransferase. Chem. Phys. Lett. 463, 214-218.

12. (a) Becke, A. D. (1993) Density-functional thermochemistry 3. The role of exact exchange. J. Chem. Phys. 98, 5648-5652. (b) Lee, C., Yang, W., and Parr, R. G. (1988) Development of the Colle-Salvetti correlation-energy formula into a functional of the electron density. Phys. Rev. B 37, 785-789.

13. Frisch, M. J., et al. (2004) Gaussian 03, revision D.01, Gaussian, Inc., Wallingford, CT.

14. (a) Barone, V., and Cossi, M. (1998) Quantum calculation of molecular energies and energy gradients in solution by a conductor solvent model. J. Phys. Chem. A 102, 1995-2001. (b) Cossi, M., Rega, N., Scalmani, G., and Barone, V. (2003) Energies, structures, and electronic properties of molecules in solution with the C-PCM solvation model. J. Comput. Chem. 24, 669-681.

15. (a) Vrček, I. V., Vrček, V., and Siehl, H.-S. (2002) Quantum Chemical Study of Degenerate Hydride Shifts in Acyclic Tertiary Carbocations. J. Phys. Chem. A 106, 1604-1611. (b) de M. Carneiro, J. W., Schleyer, P. v. R., Koch, W., and Raghavachari, K. (1990) Structure of the 2-butyl cation. Hydrogen bridged or methyl bridged? J. Am. Chem. Soc. 112, 4064 4066. (c) Fărcașiu, D., and Norton, S. H. (1997) Secondary and Tertiary 2-Methylbutyl Cations. 3. Theoretical Study of Structures and Interconversions of $\mathrm{C}_{5} \mathrm{H}_{11}{ }^{+}$Ions in the Gas Phase. J. Org. Chem. 62, 5374-5379.

16. (a) Gallivan, J. P., and Dougherty, D. A. (1999) Cation- $\pi$ interactions in structural biology. Proc. Natl. Acad. Sci. U.S.A. 96, 9459-9464. (b) Coletti, C., and Re, N. (2006) Theoretical Study of Alkali CationBenzene Complexes: Potential Energy Surfaces and Binding Energies with Improved Results for Rubidium and Cesium. J. Phys. Chem. A 110, 6563-6570. (c) Albertí, M., Aguilar, A., Lucas, J. M., Pirani, F., 
Cappelletti, D., Coletti, C., and Re, N. (2006) Atom-Bond Pairwise Additive Representation for Cation-Benzene Potential Energy Surfaces: An ab Initio Validation Study. J. Phys. Chem. A 110, 90029010.

17. Grogan, D. W., and Cronan, J. E., Jr. (1997) Cyclopropane ring formation in membrane lipids of bacteria. Microbiol. Mol. Biol. Rev. $61,429-441$.

18. Several attempts were made to locate the transition state for this proton transfer without success. A very small conformational change causes the proton to move to the bicarbonate.

19. Zhao, Y., Lynch, B. J., and Truhlar, D. G. (2004) Development and Assessment of a New Hybrid Density Functional Model for Thermochemical Kinetics. J. Phys. Chem. A 108, 2715-2719.

20. Perdew, J. P., Chevary, J. A., Vosko, S. H., Jackson, K. A., Pederson, M. R., Singh, D. J., and Fiolhais, C. (1992) Atoms, molecules, solids, and surfaces: Applications of the generalized gradient approximation for exchange and correlation. Phys. Rev. B 46, 6671-6687.

21. We also calculated the barriers for the rearrangement and the deprotonation reactions using $\mathrm{BB} 1 \mathrm{~K}$ and $\mathrm{B} 3 \mathrm{PW} 91$ (with the $6-31 G^{* *}$ basis set) for the ionized Glu140 model. The barrier difference was even smaller than with B3LYP, by 0.9 and $0.7 \mathrm{kcal} / \mathrm{mol}$, respectively.

22. (a) Li, A.-H., Dai, L.-X., and Aggarwal, V. K. (1997) Asymmetric Ylide Reactions: Epoxidation, Cyclopropanation, Aziridination, Olefination, and Rearrangement. Chem. Rev. 97, 2341-2372. (b) SolladiéCavallo, A., Diep-Vohuule, A., and Isarno, T. (1998) Two-Step
Synthesis of trans-2-Arylcyclopropane Carboxylates with $98-100 \%$ e.e. by the Use of a Phosphazene Base. Angew. Chem., Int. Ed. 37, 1689-1691. (c) Aggarwal, V. K., Alonso, E., Fang, G., Ferrara, M., Hynd, G., and Porcelloni, M. (2001) Application of chiral sulfides to catalytic asymmetric aziridination and cyclopropanation with in situ generation of the diazo compound. Angew. Chem., Int. Ed. 40, 14331436. (d) Müller, P., and Boléa, C. (2001) Carbenoid Pathways in Copper-Catalyzed Intramolecular Cyclopropanations of Phenyliodonium Ylides. Helv. Chim. Acta 84, 1093-1111. (e) Ye, S., Huang, Z.-Z., Xia, C.-A., Tang, Y., and Dai, L.-X. (2002) A Novel Chiral Sulfonium Yilde: Highly Enantioselective Synthesis of Vinylcyclopropanes. J. Am. Chem. Soc. 124, 2432-2433. (f) Aggarwal, V. K., and Grange, E. (2006) Asymmetric Sulfonium Ylide Mediated Cyclopropanation: Stereocontrolled Synthesis of (+)-LY354740. Chem.-Eur. J. 12, $568-575$.

23. (a) Aggarwal, V. K., and Winn, C. L. (2004) Catalytic, asymmetric sulfur ylide-mediated epoxidation of carbonyl compounds: scope, selectivity, and applications in synthesis. Acc. Chem. Res. 37, 611-620. (b) Gandelman, M., Naing, K. M., Rybtchinski, B., Poverenov, E., Ben-David, Y., Ashkenazi, N., Gauvin, R. M., and Milstein, D. (2005) A General Method for Preparation of Metal Carbenes via Solution- and Polymer-Based Approaches. J. Am. Chem. Soc. 127, 15265-15272. (c) Ma, M., Peng, L., Li, C., Zhang, X., and Wang, J. (2005) Highly Stereoselective [2,3]-Sigmatropic Rearrangement of Sulfur Ylide Generated through Cu(I) Carbene and Sulfides. J. Am. Chem. Soc. 127, 15016-15017. 